\title{
Design and Fabrication of Slidable Pedicle Screw Frame System for Surgery Therapy of Patients with Spinal Deformity in Grow- th Period
}

Zhenglei Wang*, Jichang Gao, Danbing Jia, Wenjing Zhang, Peng Sun, Qi Qi, Ming gong, Wenxian Fan, Xianyu Wu, Fuquan Zhao

Department of Orthopedics of No. 211 Hospital of the PLA, Harbin 150080, P. R. China

*Corresponding author: WangZhenglei211@sina.com

\begin{abstract}
With the aim of preventing iatrogenic shot trunk, stiffness and rotation deformity caused by long-term fixation with usual locked pedicle screw system, a new kind of slidable pedicle screw frame system was designed and fabricated to treat patients with spinal deformity in the growth period. The frame was composed of slidable and locked pedicle screws, cross-links and double rods. The middle part of double rods was fixed with locked pedicle screws and two ends of them were with slidable ones. The spine longitudinal and transverse growth was not limited. 360 patients including 82 congenital scoliosis, 110 idiopathic scoliosis, 60 kyphosis, 44 spinal fractures and 63 spinal tumors or tuberculosis were treated. The average preoperative Cobb's angle of scoliosis was $66.43^{\circ}$, post-operative one was $22.43^{\circ}$ and lost $5^{\circ}$ with average 14 months of following-up (6-37 months). The total longitudinal growth of adolescent spinal deformity was $86.4 \mathrm{~cm}$ (mean $0.9 \mathrm{~cm})$. In conclusion, slidable pedicle screw frame system is successfully designed and fabricated, also stable and effective to correct the spinal deformities in adolescent growth period. The longitudinal and transverse spine growth of post-operation patients was not limited, and no complications such as stiffness, vertebrae rotation and crank shaft happened for post-operation patients.
\end{abstract}

Keywords: Spinal deformity, Spinal growth, Slidable pedicle screw frame system

Citation: Z. Wang, et al. Design and Fabrication of Slidable Pedicle Screw Frame System for Surgery Therapy of Patients with Spinal Deformity in Growth Period. Nano Biomed Eng 2011; 3(2):126-129. DOI: 10.5101/nbe.v3i2.p126-129.

\section{Introduction}

Spinal abnormality surgery therapy has been done for more than twenty years in China [1]. In early time of 1980s, Harrington hook and club supporting method was intensively used, and obtained some therapeutic effects, could correct average 22 degree angle [2]. In the early time of 1990s, the method of steel-fixing-club under Luque vertebral ban could correct average 40 degree angle. In the medium of $1990 \mathrm{~s}$, no. 3 generation of spinal pedicle screw system was used for clinical therapy, displayed unique good effects, its strong fixing and three-dimension correcting abnormality achieved over $90 \%$ satisfaction ratio, correction ratio reached to over $70 \%$ [3]. However, this method exists another unignorable question: for those young patients in the periods of growth and development, the method of long spinal fragment lock-up and planted bone fusion for therapy of spinal abnormality easily lead to ankylosing spondylitis, shorten body height, seriously affect adolescent spinal growth and development [4]. Although a lot of methods were tried to solve the problem, the effects were not good or needs higher costs [5-8]. Since 2001, we designed and fabricated a set of slidable pedicle screw frame system, and used it for therapy of 360 patients with spinal deformity in the period of growth and development, and followed up 96 post-operative patients, acquired good therapeutic effects.

\section{Experimental Section}

\subsection{Clinical Data}

From 2001 to 2006,360 patients with spinal deformation including 82 cases of congenital spinal sidebends, 110 cases of special spinal side-bends, of which included 23 cases of unplanted bones, 60 cases of spinal 
rear projecting, 44 cases of spinal fractures, 63 cases of spinal tumors or tuberculosis. Patients with side-bends and rear-projecting had average curve degree of 66.43 .

\subsection{Design and fabrication of slidable pedicle screw frame system}

According to our clinical experiences and patients' requirement, we designed the slidable pedicle screw frame system, and applied its patent. This system including every device were fabricated with titanic alloy materials.

\subsection{Operation Method}

\subsubsection{Anaesthesia and spinal bone exposure}

Patients were anaesthetized, controllable low blood pressure, shrink pressure was controlled within $90 \mathrm{~mm}$ $\mathrm{Hg}$, monitoring induced potentials. Rear medium incision, skin was cut and open, electronic knife cut subcuateous tissues and deep tissues up to thorn upper ligament, twoside spinal bans were exposed, fully exposed operational field.

\subsubsection{Allocation of spinal body nail}

Firstly, to ensure nailed spinal body in main bend (2-3 spinal bodies), that is, two spinal bodies in upper spinal were set up 4 locked nails, in each end of spinal bodies, set up one slidable nail between every two spinal bodies, averagely set 4-6 nails at each end of spinal body. The setting nail method: hand-drill dig into spinal body sidefrontage through the rear of spinal bow root, the deep length is 3 to $4 \mathrm{~cm}$. First nail is orientated by using $\mathrm{C}$ type $\mathrm{X}$-ray instrument, to measure the position of spinal body and angle and deep length of nails, and then drill all nails into spinal bow roots, in order to ensure safety, nails were drilled into spinal body under the monitoring of induced potentials.

\subsubsection{Allocation of spinal root screw frame}

Spinal pedicle screw' termination faced to internal side, identical length correcting clubs with over $3 \mathrm{~cm}$ compared with upper and down terminals, bending device was bent to physiological curve, from upper to down press nails into right position, $U$ type cap was filled into the termination, screw caps got down,after two clubs were fixed, used circle-club devices slowly screw two clubs, until spinal deformity was corrected satisfactorily, then used 4 locked nails to fix two clubs, finally put transverse orientation locked ligation poles, the patients with serious deformity were set up locked longitudinal ligation poles, locked the nails, enhancing the fixed length, frame structure was finished.

\subsubsection{Planted bone fusion}

http://nanobe.org

For patients with inherited spinal side-bend, special spinal side-bend, and cut-bone-correction position, in their locked nail scope, regular planted bone, slide-nail area could not plant bones. Cleaning soft tissues, and cut cortex bone into scale shapes, pick up rib from deform area, cut it into thin strips and added into artificial bones and fused them together, for the patients with Cobb's angle of less than 40 degree and spinal sections with good tenacity, did not need planted bone and fusion.

\subsubsection{Incision suture}

We used physiological salt water to wash the incision, stanched completely, and then sprayed suitable amounts of antibiotics and dexamethasone mixtures, sutured layer by layer, patients were kept on their back at postoperation.

\subsection{Results and Discussion}

\subsubsection{Design and fabrication of slidable pedicle screw frame system}

As shown in Fig. 1, the slidable pedicle screw

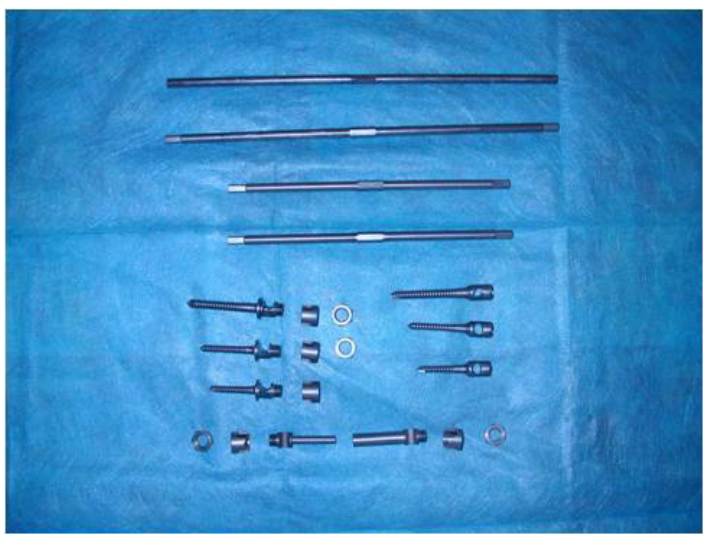

Fig. 1 Picture of actual unlocked structures such as pedicle screws, club and rods

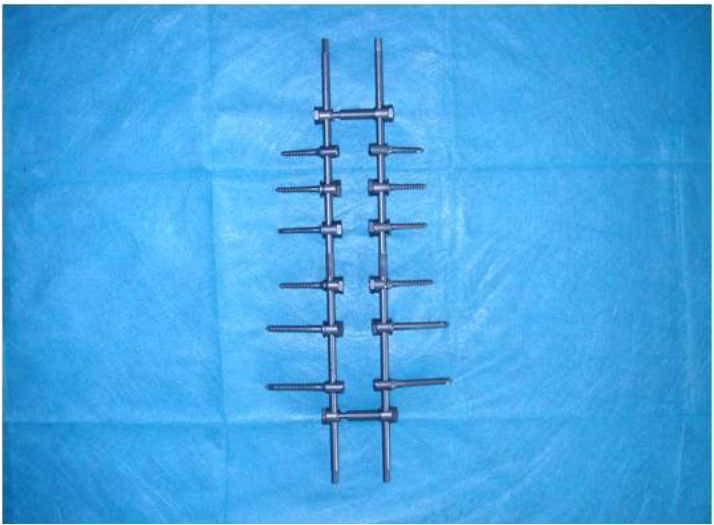

Fig. 2 Picture of actual part locked structures such as pedicle screws, club and cross-linked double rods 
appear those signs such as broken nails, broken clubs and nerve damages. structures such as pedicle screws, clubs and crosslink rods. These devices were made with titanic alloy materials. Fig. 2 showed actual part locked structures such as pedicle screws, club and cross-linked double rods, which was used in the clinical therapy.

\subsubsection{Clinical therapeutic effects analysis}

As shown in Fig. 3, all detail devices were displayed. As shown in Fig. 4, the frame structure system was used in clinical patients' therapy. From 2001 to 2006, we treated 360 patients with different spinal abnormality, including 82 cases of congenital spinal side-bends, 110 cases of special spinal side-bends, which of them included 23 cases of unplanted bones, 60 cases of spinal rear projecting, 44 cases of spinal fractures, 63 cases of spinal tumors or tuberculosis. Patients with side-bends and rear-projecting had average curve degree of 66.43 , post-operation patients with average 22.43 degree, correction ratio is $66.24 \%$, of which for the patients with Cobb's angle of less than or equal to 50 degree, the correction ratio is $80 \%$. In the period of 12 to 36 months post-operation, 96 patients were followed up, longitudinal growth is $86.4 \mathrm{~cm}$ in length, spinal of fixed section scope grew $0.9 \mathrm{~cm}$, correction lose 5 degree, all patients did not

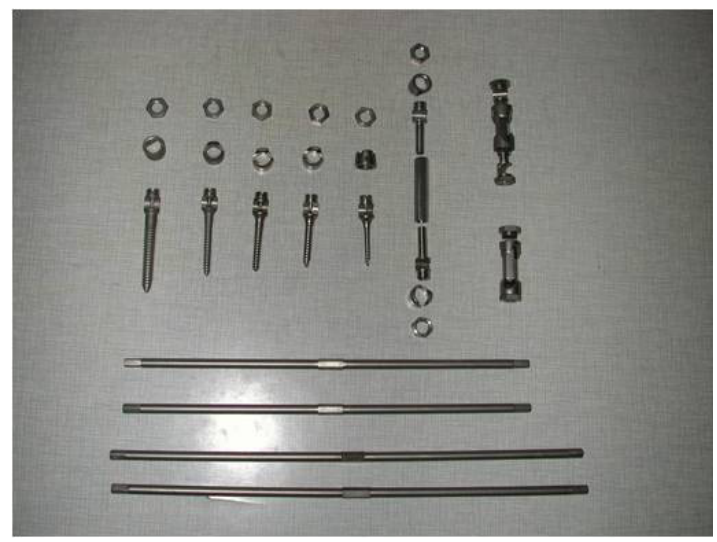

Fig. 3 Picture of part locked structure such as pedicle screws, clubs, transverse cross-link poles

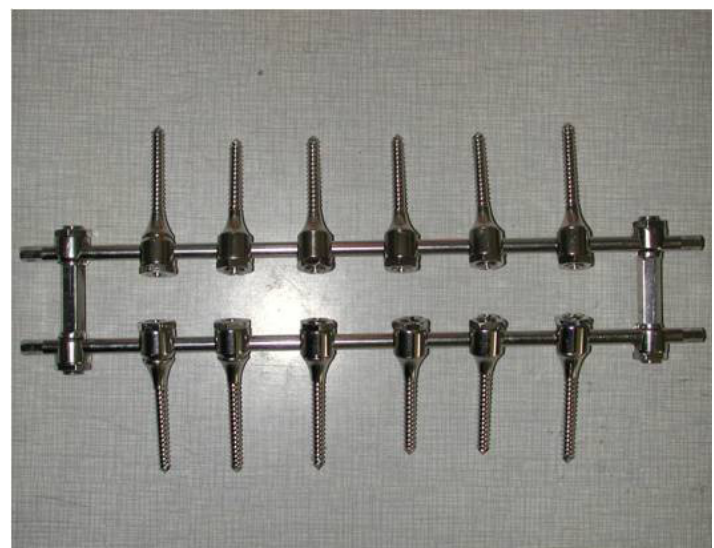

Fig. 4 Picture of locked frame structure such as pedicle screws, clubs, transverse cross-link rods

\subsection{Therapeutic effects analysis}

Spinal abnormality surgery therapy has existed for more than twenty years in China. However, traditional therapeutic methods still exist some serious problems, for example, correction effects is not good, causing serious side-bend, and enhanced axis-directed deformity. In recent ten years, foreign countries' scholars used no. 3 generation vertebra bow pedicle screw therapy technology to treat spinal deformity and achieved better clinical effects [2-5], and carefully investigated fixed method, position, and planted bone fusion scope. Compared with previous Harrington and Luque's methods, new method owns marked advantages [6]. In the course of treating spinal deformity, suitable operation method and inner fixation devices are very important, and all those operations can not affect spinal's growth and development [7-13].

Rapid growth period of children and the young is considered to be risk periods for spinal deformity [4, $14]$, at the ages of 8 to 16 years old, children happened diseases more early, this kind of serious disease' degree will become more obviously. So far in china, intensive usage's nail club ligation system is locked correction fixation. Fixing vertebra body, locking nails fixation and planted bone fusion, which cause limited spinal growth, which does not fit for human body physiological development rule $[14,15]$. Hefti and chaster introduced curved axis growth phenomena of spinal fusion in the ages of 8-11 years old, which is because inner fixation is not suitable $[8,15]$. In order to avoid the curved axis growth phenomena,some new methods were tried, however, long term effects are not clear [3, 7-13].

Since 2001, we used slidable pedicle screw frame system to correct spinal side bend, results showed that ratio is $66.24 \%$, for those patients with Cobb's angle less than or equal to 50 degree, their correction ratio is $80 \%$, this method is very successful, avoid syndromes such as correction lose, and fake joint formation. For those patients with serious structure spinal side-bend, and bone loosen symptom, intervertebral cartilages metamorphosis, did not need to recover completely normal spinal physiological curve degree, avoid radix arcus vertebrae broken, spinal un-balance, spinal cord damage, etc. For those patients with un-structure side-bend or slight special children side-bend (Cobb's angle $\leq 40$ degree), correct deformity by using inner fixation system, did not need planted bone fusion, with the aim of decreasing limited spinal action, back pain and curved axis phenomena. For those patients with congenital spinal side-bend and other spinal diseases'pathological abnormality, etc., it is necessary to use rear-column lot planted bone fusion this method is very effective for all patients, corrdction 
method to enhance post-spinal stability.

Although prepared slidable pedicle screw frame system is used to treat spinal deformity and achieve good effects, there still exist some questions, for example, the whole system is composed of titanic alloy materials, titanic alloy materials' quality is very important, affects therapeutic effects. Up to date, its long term therapeutic effects is still not clarified well [16]. As the nanotechnology rapidly develops, a lot of nanomaterials with unique structure and function are fabricated ceaselessly [17], especially biodegradable nanomaterials are fabricated, which bring great chance to design and fabricate novel slidable pedicle screw frame system, which will provide more suitable devices for therapy of spinal deformity in near future.

\section{Conclusion}

A new kind of slidable pedicle screw frame system composed of slidable and locked pedicle screws, crosslinks and double rods was successfully designed and fabricated and used to treat patients with spinal deformity in the growth period, and achieved good therapeutic effects, this system owns broad application prospect in therapy of spinal deformity in near future.

\section{References}

1. Harrington PR, Dichson JH. Spinal Instrumentation in the severe progressive spondlo listhesis. Clin Orthop.1976; 117-157.

2. Raymond Roy-camile.et al.internal fixation of the lumbar spine with pedicle screw plating. Clin Orthop. 1986; 203: 7

3. Li SG, Sheng L, Zhao H, Zhang JG, Zhai JL, Zhu Y. Computer-assisited navigation technique in the spinal pedicle screw internal fixation. Journal of Clinical Rehabilitative Tissue Engineering Research 2009; 13(7): 3365-3369.

4. Qiu GX. Spinal side-convex therapy. Chinese Journal of Orthopaedics 1995; 15( 0): 15-717.

5. Hu YG, Qiu GX, Tang TS. Spinal surgery. People Sanitation Press 2000; no 2 version, p377.

6. Ye QB, Qiu GX, Wang YM. Early results of PRSS' therapy for spi- nal side bend of children and the young in the course of growth and development. Orthopedic Journal of China 2001; 8(1): 16-20.

7. Wen XS, Jiu GX, Zhang J. Long term therapeutic effects evaluation of inner fixation of spinal Pedicle. Chinese Journal of Orthopaedics 2001; 21(11): 64-665.

8. Dick W, et al. A new device for internal fixation of thoraco-lumbar and lumbar spine fracture: the fixation in terne. Paraplegia.1959; 23 $: 225$.

9. Hakalo J, Wroński J. Complications of a transpedicular stabilization of thoraco-lumbar burst fractures. Neurol Neurochir Pol. 2006; 40: 134-139.

10. Fuentes S, Blondel B, Metellus P, Gaudart J, Adetchessi T, Dufour H.Percutaneous kyphoplasty and pedicle screw fixation for the management of thoraco-lumbar burst fractures. Eur Spine J. 2010; 19(8):1281-1287. doi:10.1007/s00586-010-1444-4

11. Steib JP, Mezghani S, Charles YP, Mitulescu A. Double approach in thoraco-lumbar malunions. Eur Spine J 2010; Suppl 1:S48-51. doi: $10.1007 / \mathrm{s} 00586-009-1165-8$

12. Arregui R, Martínez-Quiñones JV, Aso-Escario J, Aso-Vizan J. Vertebral reinforcement by means of kyphoplasty in the treatment of non-osteoporotic thoraco-lumbar fractures. Study of 40 cases and review of the literature. Neurocirugia (Astur). 2008; 19(6): 537-5350.

13. Fuentes S, Metellus P, Fondop J, Pech-Gourg G, Dufour H, Grisoli F. Percutaneous pedicle screw fixation and kyphoplasty for management of thoracolumbar burst fractures. Neurochirurgie. 2007; 53(4): 272-276. doi:10.1007/s00701-005-0681-5

14. Payer M. Unstable burst fractures of the thoraco-lumbar junction: treatment by posterior bisegmental correction/fixation and staged anterior corpectomy and titanium cage implantation. Acta Neurochir (Wien). 2006; 148(3): 299-306. doi:10.1007/s00701-005-0681-5

15. Stulík J, Krbec M, Vyskocil T. Use of bioceramics in the treatment of fractures of the thoraco-lumbar spine. Acta Chir Orthop Traumatol Cech. 2002; 69(5): 288-94.

16. Andress HJ, Braun H, Helmberger T, Schürmann M, Hertlein H, HartlWH. Long-term results after posterior fixation of thoracolumbar burst fractures. Injury. 2002; 33(4): 357-365.doi:10.1016/S0 020-1383(02)00030-X

17. Changxiao Liu. Research and development of nanopharmaceuticals in China. Nano Biomed Eng 2009; 1(1):1-12. doi:10.5101/nbe.v1i1. p1-12

Copyright:(c) $2011 \mathrm{Z}$. Wang, et al. This is an openaccess article distributed under the terms of the Creative Commons Attribution License, which permits unrestricted use, distribution, and reproduction in any medium, provided the original author and source are credited. 